\title{
On a dimension formula for spherical twisted conjugacy classes in semisimple algebraic groups
}

\author{
Jiang-Hua Lu
}

Received: 2 May 2010 / Accepted: 11 September 2010 / Published online: 30 September 2010

(C) The Author(s) 2010. This article is published with open access at Springerlink.com

\begin{abstract}
Let $G$ be a connected semisimple algebraic group over an algebraically closed field of characteristic zero, and let $\theta$ be an automorphism of $G$. We give a characterization of spherical $\theta$-twisted conjugacy classes in $G$ by a formula for their dimensions in terms of certain elements in the Weyl group of $G$, generalizing a result of N. Cantarini, G. Carnovale, and M. Costantini when $\theta$ is the identity automorphism. For $G$ simple and $\theta$ an outer automorphism of $G$, we also classify the Weyl group elements that appear in the dimension formula.
\end{abstract}

\section{Introduction}

\subsection{The main results}

If $R$ is a group and $\theta$ is an automorphism of $R$, the $\theta$-twisted conjugation of $R$ on itself is defined by $r \cdot{ }_{\theta} r^{\prime}=r r^{\prime} \theta(r)^{-1}$ for $r, r^{\prime} \in R$, and its orbits are called the $\theta$-twisted conjugacy classes in $R$.

Let $G$ be a connected semisimple algebraic group over an algebraically closed field $\mathbf{k}$ of characteristic zero, and let $\operatorname{Aut}(G)$ be the automorphism group of $G$. For $\theta \in \operatorname{Aut}(G)$, a $\theta$-twisted conjugacy class $C$ in $G$ is said to be spherical if a Borel subgroup of $G$ has an open orbit in $C$ for the $\theta$-twisted conjugation action.

Fix a Borel subgroup $B$ of $G$ and a maximal torus $H \subset B$, and let $\operatorname{Aut}^{\prime}(G)=\{\theta \in$ $\operatorname{Aut}(G): \theta(B)=B, \theta(H)=H\}$. Throughout the paper, we assume that $\theta \in \operatorname{Aut}^{\prime}(G)$ (see Remark 1.2).

Let $W=N_{G}(H) / H$ be the Weyl group, where $N_{G}(H)$ is the normalizer of $H$ in $G$, and let $l$ be the length function on $W$. For $w \in W$, denote by $\operatorname{rk}(1-w \theta)$ the rank of the linear operator $1-w \theta$ on the Lie algebra $\mathfrak{h}$ of $H$. For a $\theta$-twisted conjugacy class $C$ in $G$, let $m_{C}$ be

J.-H. Lu (凶)

Department of Mathematics, Hong Kong University, Pokfulam Rd., Hong Kong, Hong Kong e-mail: jhlu@maths.hku.hk 
the unique element in $W$ such that $C \cap\left(B m_{C} B\right)$ is dense in $C$. In the first part of the paper, we prove the following characterization of spherical $\theta$-twisted conjugacy classes in $G$.

Theorem 1.1 For $\theta \in \operatorname{Aut}^{\prime}(G)$, a $\theta$-twisted conjugacy class $C \subset G$ is spherical if and only if

$$
\operatorname{dim} C=l\left(m_{C}\right)+\operatorname{rk}\left(1-m_{C} \theta\right) .
$$

When $\theta=\operatorname{Id}_{G}$, the identity automorphism of $G$, Theorem 1.1 is proved by Cantarini etal. in [1] by a case-by-case checking that depends on the classification of all spherical conjugacy classes in $G$ (for $G$ simple). Formula (1.1) is then used in [1] to prove the De Concini-Kac-Procesi conjecture on representations of the quantized enveloping algebra of $G$ at roots of unity over spherical conjugacy classes. A different proof of Theorem 1.1 for $\theta=\operatorname{Id}_{G}$, which is also valid when the characteristic of $\mathbf{k}$ is an odd good prime for $G$, is given by Carnovale in [2], where the proof does not require a classification of spherical conjugacy classes in $G$ but it also depends on some case-by-case computations. When $\theta^{2}=\operatorname{Id}_{G}$ and $C$ is the $\theta$-twisted conjugacy class through the identity element of $G,(1.1)$ follows from standard results on symmetric spaces (see §2.3).

In $\S 2$, we give a direct proof of Theorem 1.1.

For $\theta=\operatorname{Id}_{G}$, the elements $m_{C}$ play an important role in the study of spherical conjugacy classes. In particular, it is shown by Costantini [5] that the coordinate ring of a spherical conjugacy class $C$ as a $G$-module is almost entirely determined by $m_{C}$ (see [5, Theorem 3.22]). For $G$ simple and of classical type and for $\theta=\operatorname{Id}_{G}$, the element $m_{C}$ for every conjugacy class in $G$ is computed explicitly in [4]. The second part of the paper concerns the set

$$
\widetilde{\mathcal{M}}_{\theta}=\left\{m_{C}: C \text { is a } \theta \text {-twisted conjugacy class in } G\right\} \subset W
$$

for an arbitrary $\theta \in \operatorname{Aut}^{\prime}(G)$. The same arguments used in the proof of [3, Remark 2] show that the set $\widetilde{\mathcal{M}}_{\theta}$ depends only on the isogeny class of $G$. Denote also by $\theta$ the automorphism of $W$ naturally induced from $\theta \in \operatorname{Aut}^{\prime}(G)$ (see $\$ 3.1$ for more detail), and let

$$
\begin{array}{r}
\mathcal{M}_{\theta}=\{m \in W: \quad m \text { is the unique maximal length element } \\
\text { in its } \theta \text {-twisted conjugacy class in } W\},
\end{array}
$$

By[3, Corollary 2.15], $\widetilde{\mathcal{M}}_{\theta} \subset \mathcal{M}_{\theta}$.

For $G$ simple and $\theta$ an inner automorphism of $G$, it is shown in [3, §3] that $\widetilde{\mathcal{M}}_{\theta}=\mathcal{M}_{\theta}$ and elements in $\mathcal{M}_{\theta}$ are classified in [3, §3] using results from [1,2]. For $G$ simple and $\theta$ an outer automorphism of $G$, we give in Proposition 3.7 the complete list of elements in $\mathcal{M}_{\theta}$, and we prove in Theorem 3.8 that, again, $\widetilde{\mathcal{M}}_{\theta}=\mathcal{M}_{\theta}$. It turns out that if $\theta$ induces an order 2 automorphism of the Dynkin diagram of $G$, the list of elements in $\mathcal{M}_{\theta}$ coincides with that of Springer in [10, Table 2], and if $G=D_{4}$ and $\theta$ has order 3, $\mathcal{M}_{\theta}$ has two elements. The classification of elements in $\widetilde{\mathcal{M}}_{\theta}$ gives restrictions on the possible dimensions of $\theta$-twisted conjugacy classes in $G$. See Example 3.9.

\subsection{Notation}

Let $\Delta_{+}$and $\Gamma \subset \Delta_{+}$be respectively the sets of positive and simple roots determined by $(B, H)$ and write $\alpha>0$ (resp. $\alpha<0$ ) for $\alpha \in \Delta_{+}$(resp. $\alpha \in-\Delta_{+}$). Let $N$ and $N_{-}$be respectively the unipotent radicals of $B$ and the opposite Borel subgroup $B_{-}$. The Lie algebras of $G, B, H, N$, and $N_{-}$are respectively denoted by $\mathfrak{g}, \mathfrak{b}, \mathfrak{h}, \mathfrak{n}$, and $\mathfrak{n}_{-}$. For $\alpha>0, s_{\alpha}$ denotes the corresponding reflection in $W$. We also fix a representative $\dot{w}$ in $N_{G}(H)$ for each $w \in W$. 
For $\theta \in \operatorname{Aut}^{\prime}(G)$, we use the same letter to denote the action of $\theta$ on $\Delta_{+}$, and when necessary, we write $\theta \in \operatorname{Aut}(\Gamma)$ to indicate that $\theta$ is regarded as an automorphism of the Dynkin diagram. The induced action of $\theta$ on $\mathfrak{g}$ is also denoted by $\theta$.

For $g \in G, \operatorname{Ad}_{g}$ denotes both the conjugation on $G$ by $g$ and the induced map on $\mathfrak{g}$. For a set $V$ and a map $\sigma: V \rightarrow V$, we let $V^{\sigma}=\{x \in V: \sigma(x)=x\}$.

Remark 1.2 For an arbitrary $\theta_{1} \in \operatorname{Aut}(G)$, there exists $g_{0} \in G$ such that $\operatorname{Ad}_{g_{0}}(B)=\theta_{1}(B)$ and $\operatorname{Ad}_{g_{0}}(H)=\theta_{1}(H)$. Then $\theta=\operatorname{Ad}_{g_{0}}^{-1} \circ \theta_{1} \in \operatorname{Aut}^{\prime}(G)$, and the right translation by $g_{0}$ in $G$ maps $\theta_{1}$-twisted conjugacy classes in $G$ to $\theta$-twisted conjugacy classes in $G$. We can thus assume throughout the paper that $\theta \in \operatorname{Aut}^{\prime}(G)$. Moreover, if $\theta$ and $\theta^{\prime} \in \operatorname{Aut}^{\prime}(G)$ are in the same inner class, i.e., if they induce the same automorphism on the Dynkin diagram, then $\theta=\operatorname{Ad}_{h} \circ \theta^{\prime}$ for some $h \in H$, and it follows that $\widetilde{\mathcal{M}}_{\theta}=\widetilde{\mathcal{M}}_{\theta^{\prime}}$.

\section{Proof of Theorem 1.1}

\subsection{Two lemmas on $B$-orbits in $G$}

Recall that ${ }_{\theta} \theta$ denotes the $\theta$-twisted conjugation action of $G$ on itself. For $g \in G$, let $B_{g}$ be the stabilizer subgroup of $B$ at $g$. The following generalization of [1, Theorem 5] is proved in [6, Theorem 4.1]. We include the (short) proof for the convenience of the reader and to make the proof of Theorem 1.1 self-contained.

Lemma 2.1 [6] For any $w \in W$ and $g \in w B$, one has $B_{g} \subset H^{w \theta}\left(N \cap \operatorname{Ad}_{\dot{w}}(N)\right)$. Consequently,

$$
\operatorname{dim}(B \cdot \theta g) \geq l(w)+\operatorname{rk}(1-w \theta) .
$$

Proof Let $b=n_{1} n_{2} h \in B_{g}$, where $h \in H, n_{1} \in N \cap \operatorname{Ad}_{\dot{w}}\left(N_{-}\right)$and $n_{2} \in N \cap \operatorname{Ad}_{\dot{w}}(N)$. It follows from $b g=g \theta(b)$ and the unique decomposition $B w B=\left(N \cap \operatorname{Ad}_{\dot{w}}\left(N_{-}\right)\right) \dot{w} B$ that $n_{1}=1$ and $w \theta(h)=h$. Thus $B_{g} \subset H^{w \theta}\left(N \cap \operatorname{Ad}_{\dot{w}}(N)\right)$, and

$$
\begin{aligned}
\operatorname{dim}(B \cdot \theta g) & =\operatorname{dim} B-\operatorname{dim} B_{g} \geq \operatorname{dim} B-\operatorname{dim}\left(N \cap \operatorname{Ad}_{\dot{w}}(N)\right)-\operatorname{dim} H^{w \theta} \\
& =l(w)+\operatorname{rk}(1-w \theta) .
\end{aligned}
$$

Lemma 2.2 If $w \in W$ and $g \in w B$ are such that $B \cdot_{\theta} g$ is open in $G \cdot_{\theta} g$, then $B_{g}$ is an open subgroup of $H^{w \theta}\left(N \cap \operatorname{Ad}_{\dot{w}}(N)\right)$.

Proof Let $\mathfrak{g}_{g}=\left\{x \in \mathfrak{g}: \operatorname{Ad}_{g} \theta(x)=x\right\}$ be the stabilizer subalgebra of $\mathfrak{g}$ at $g$ for the $\theta$-twisted conjugation action, and let $\mathfrak{b}_{g}=\mathfrak{b} \cap \mathfrak{g}_{g}$. By Lemma 2.1, $\mathfrak{b}_{g} \subset \mathfrak{h}^{w \theta}+\left(\mathfrak{n} \cap \operatorname{Ad}_{\dot{w}}(\mathfrak{n})\right)$. It remains to prove that $\mathfrak{h}^{w \theta}+\left(\mathfrak{n} \cap \operatorname{Ad}_{\dot{w}}(\mathfrak{n})\right) \subset \mathfrak{b}_{g}$.

Let $x_{0} \in \mathfrak{h}^{w \theta}$ and $x_{+} \in \mathfrak{n} \cap \operatorname{Ad}_{\dot{w}}(\mathfrak{n})$, and let $z=\left(\operatorname{Ad}_{g} \theta\right)^{-1}\left(x_{+}+x_{0}\right)-\left(x_{+}+x_{0}\right)$ so that $\operatorname{Ad}_{g} \theta\left(z+x_{+}+x_{0}\right)=x_{+}+x_{0}$. Using the fact that $\operatorname{Ad}_{b}\left(x_{0}\right)-x_{0} \in \mathfrak{n}$ for any $b \in B$, one sees that $z \in \mathfrak{n}$. We now show that $z=0$. To this end, let $\langle$,$\rangle be the Killing form of \mathfrak{g}$. Since $B \cdot{ }_{\theta} g$ is open in $G \cdot{ }_{\theta} g$, the inclusion $\mathfrak{b} \hookrightarrow \mathfrak{g}$ induces an isomorphism $\mathfrak{b} / \mathfrak{b}_{g} \cong \mathfrak{g} / \mathfrak{g}_{g}$. Thus for any $y \in \mathfrak{g}$, there exists $y^{\prime} \in \mathfrak{b}$ such that $y-y^{\prime} \in \mathfrak{g}_{g}$, and, using $\left\langle z, y^{\prime}\right\rangle=0$, one has

$$
\begin{aligned}
\langle z, y\rangle & =\left\langle z+x_{+}+x_{0}, y-y^{\prime}\right\rangle-\left\langle x_{+}+x_{0}, y-y^{\prime}\right\rangle \\
& =\left\langle z+x_{+}+x_{0}, y-y^{\prime}\right\rangle-\left\langle\operatorname{Ad}_{g} \theta\left(z+x_{+}+x_{0}\right), \operatorname{Ad}_{g} \theta\left(y-y^{\prime}\right)\right\rangle=0 .
\end{aligned}
$$

It follows that $z=0$ and hence $x_{+}+x_{0} \in \mathfrak{b}_{g}$. Therefore $\mathfrak{b}_{g}=\mathfrak{h}^{w \theta}+\left(\mathfrak{n} \cap \operatorname{Ad}_{\dot{w}}(\mathfrak{n})\right)$. 


\subsection{Proof of Theorem 1.1}

Let $C$ be a $\theta$-twisted conjugacy class in $G$. Assume first that $\operatorname{dim} C=l\left(m_{C}\right)+\operatorname{rk}\left(1-m_{C} \theta\right)$. By Lemma 2.1, every $B$-orbit in $C \cap\left(B m_{C} B\right)$ is open in $C$, so $C$ is spherical. Since $C$ is irreducible, it also follows that $C \cap\left(B m_{C} B\right)$ is a single $B$-orbit.

Assume that $C$ is spherical. Let $g \in C$ be such that $B \cdot{ }_{\theta} g$ is open in $C$, and let $g \in B w B$ with $w \in W$. Then $C \cap(B w B) \supset B \cdot \theta g$ is dense in $C$, so $w=m_{C}$. By Lemma 2.2, $\operatorname{dim} C=\operatorname{dim} \mathfrak{b}-\operatorname{dim} \mathfrak{b}_{g}=l\left(m_{C}\right)+\operatorname{rk}\left(1-m_{C} \theta\right)$. This finishes the proof of Theorem 1.1.

Remark 2.3 For $\theta=\mathrm{Id}_{G}$, Lemma 2.2 is also proved in [2] by some case-by-case arguments. On the other hand, the arguments in [2] are valid when the characteristic of $\mathbf{k}$ is an odd good prime for $G$, while our proof of Lemma 2.2 is valid when the Killing form of $\mathfrak{g}$ is non-degenerate and when one has the identifications of tangent spaces $T_{g}(B \cdot \theta g) \cong \mathfrak{b} / \mathfrak{b}_{g}$ and $T_{g}\left(G \cdot{ }_{\theta} g\right) \cong \mathfrak{g} / \mathfrak{g}_{g}$, which hold when $\mathbf{k}$ is of characteristic zero.

\subsection{The case of symmetric spaces}

Assume that $\theta \in \operatorname{Aut}^{\prime}(G)$ is an involution, and let $K=G^{\theta}$ be the fixed point subgroup of $\theta$ in $G$. Then the $\theta$-twisted conjugacy class $C$ of the identity element of $G$ is isomorphic to the symmetric space $G / K$, and it is well-known [9] that $G / K$ is spherical. In this case, formula (1.1) for the dimension of $G / K$ follows from results in [9]. Indeed, using the notation in [9, §5], let $v^{o}$ be the unique open $B$-orbit in $G / K$ and let $w^{o}=\phi\left(v^{o}\right) \in W$. Then $w^{o}=m_{C}$, and it is easy to see from [9, Corollary 4.9] that $\operatorname{dim} G / K=\frac{1}{2} \operatorname{Card}\left(C_{v^{o}}^{\prime \prime}\right)+\operatorname{Card}\left(I_{v^{o}}^{n}\right)+l\left(w^{o}\right)+\operatorname{rk}\left(1-w^{o} \theta\right)$, where the notation is as on [9, Page 535]. By [9, Theorem 5.2(i)], $C_{v^{o}}^{\prime \prime} \cap \Gamma=\emptyset$. For every $\beta>0$, writing $\beta=\beta_{1}+\beta_{2}$, where $\beta_{1}$ is in the linear span of $\Pi \subset \Gamma$ in the notation of [9, Theorem 5.2(ii)] and $\beta_{2}$ is in the linear span of $\Gamma \backslash \Pi$, one has $w^{o} \theta(\beta)=\beta_{1}+w^{o} \theta\left(\beta_{2}\right)$, so by [9, Theorem 5.2(ii)], $w^{o} \theta(\beta)>0$ implies that $\beta_{2}=0$ and thus $w^{o} \theta(\beta)=\beta$. This shows that $C_{v^{o}}^{\prime \prime}=\emptyset$ and that every $\beta \in I_{v^{o}}$ is in the linear span of $\Pi$, which, by [9, Theorem 5.2(i)], consists of all simple compact imaginary roots. It follows that $I_{v^{o}}^{n}=\emptyset$. Thus $\operatorname{dim} G / K=l\left(w^{o}\right)+\operatorname{rk}\left(1-w^{o} \theta\right)$.

\section{The elements $m_{C}$}

\subsection{Properties of $m \in \mathcal{M}_{\theta}$}

Any $\delta \in \operatorname{Aut}(\Gamma)$ induces an automorphism on the Weyl group $W$, also denoted by $\delta$, by $\delta(w)=\delta \circ w \circ \delta^{-1}: \mathfrak{h} \rightarrow \mathfrak{h}$. Let $w_{0}$ be the longest element in $W$, and let $\delta_{0} \in \operatorname{Aut}(\Gamma)$ be given by $\delta_{0}(\alpha)=-w_{0} \alpha$ for $\alpha \in \Gamma$. The automorphism on $W$ induced by $\delta_{0}$ is then given by $\delta_{0}(w)=w_{0} w w_{0}$ for $w \in W$.

Throughout this section, $\theta \in \operatorname{Aut}(\Gamma)$, and $\mathcal{M}_{\theta} \subset W$ is defined as in (1.3).

Lemma 3.1 If $m \in \mathcal{M}_{\theta}$, then $\theta(m)=\delta_{0}(m)=m$. In particular, $m \theta(\alpha)=\theta m(\alpha)$ for every $\alpha \in \Gamma$.

Proof Let $m \in \mathcal{M}_{\theta}$. Then $\theta(m)=m^{-1} m \theta(m)$ is in the same $\theta$-twisted conjugacy class as $m$, and $l(\theta(m))=l(m)$. Thus $\theta(m)=m$. It follows that $m \theta(\alpha)=\theta m(\alpha)$ for every $\alpha \in \Gamma$. Similarly, since $\theta$ permutes the simple roots, $\theta\left(w_{0}\right)=w_{0}$. It follows that $w_{0} m w_{0}$ and $m$ are in the same $\theta$-twisted conjugacy class in $W$. Since $l\left(w_{0} m w_{0}\right)=l(m)$, one has $w_{0} m w_{0}=m$. 
For $I \subset \Gamma$, let $w_{0, I}$ be the longest element in the subgroup $W_{I}$ of $W$ generated by $I$.

The following Lemma 3.2 is proved in $[3, \S 3]$ when $\theta$ is the identity automorphism of $\Gamma$.

Lemma 3.2 If $m \in \mathcal{M}_{\theta}$, then $w_{0} m=m w_{0}=w_{0, I}$, where $I=\{\alpha \in \Gamma: m \theta(\alpha)=\alpha\}$. In particular, $I$ is both $\delta_{0}$ and $\theta$ invariant, and $\delta_{0} \theta(\alpha)=-w_{0, I}(\alpha)$ for every $\alpha \in I$.

Proof Let $\delta=\delta_{0} \theta \in \operatorname{Aut}(\Gamma)$. Then the map $W \rightarrow W: w \mapsto w w_{0}$ maps $\theta$-twisted conjugacy classes in $W$ to $\delta$-twisted conjugacy classes in $W$.

Let $m \in \mathcal{M}_{\theta}$, and let $x=m w_{0}$. Then $x$ is the unique minimal length element in its $\delta$-twisted conjugacy class in $W$. Let $x=s_{\alpha_{1}} s_{\alpha_{2}} \cdots s_{\alpha_{k}}$ be a reduced word for $x$. Let $I^{\prime}=$ $\left\{\alpha_{1}, \alpha_{2}, \ldots, \alpha_{k}\right\}$. Then $x \in W_{I^{\prime}}$. We first show that $x=w_{0, I^{\prime}}$. To this end, it is enough to show that $x\left(\alpha_{j}\right)<0$ for every $1 \leq j \leq k$. Since $x s_{\alpha_{k}}<x$, we already know that $x\left(\alpha_{k}\right)<0$. If $k=1$, we are done. Suppose that $k \geq 2$. Let $\beta_{k}=\delta^{-1}\left(\alpha_{k}\right) \in \Gamma$, and let

$$
x_{1}=s_{\beta_{k}} x \delta\left(s_{\beta_{k}}\right)=s_{\beta_{k}} x s_{\alpha_{k}}=s_{\beta_{k}} s_{\alpha_{1}} \cdots s_{\alpha_{k-1}} .
$$

Since $k$ is the minimal length of elements in the $\delta$-twisted conjugacy class of $x$ in $W$, we have $l\left(x_{1}\right) \geq k$. It follows from (3.1) that $l\left(x_{1}\right) \leq k$, so $l\left(x_{1}\right)=k$. Since $x$ is the unique element in its $\delta$-twisted conjugacy class in $W$ with length $k$, we have $x_{1}=x$. In particular, $x=x_{1}=s_{\beta_{k}} s_{\alpha_{1}} \cdots s_{\alpha_{k-1}}$ is a reduced word for $x$, so $x\left(\alpha_{k-1}\right)<0$. Repeating this process, we see that $x\left(\alpha_{j}\right)<0$ for every $1 \leq j \leq k$. Thus $x=w_{0} m=m w_{0}=w_{0, I^{\prime}}$. It follows from Lemma 3.1 that $\delta_{0}\left(I^{\prime}\right)=\theta\left(I^{\prime}\right)=I^{\prime}$.

We now show that $I^{\prime}=I$. For any $\alpha \in I^{\prime}$, since $m(\alpha)=w_{0} w_{0, I^{\prime}}(\alpha)>0$, one has $l\left(\theta^{-1}\left(s_{\alpha}\right) m s_{\alpha}\right) \geq l(m)$. Since $m \in \mathcal{M}_{\theta}$, one has $\theta^{-1}\left(s_{\alpha}\right) m s_{\alpha}=m$, so $\theta^{-1}(\alpha)=m(\alpha)$ and $\alpha \in I$. Conversely, let $\alpha \in I$. If $\alpha \notin I^{\prime}$, then $w_{0} m(\alpha)=w_{0, I^{\prime}}(\alpha)>0$, so $m(\alpha)<0$, contradicting the fact that $m(\alpha)=\theta^{-1}(\alpha)>0$. Thus $I^{\prime}=I$. It follows from the definition of $I$ that $\delta_{0} \theta(\alpha)=-w_{0, I}(\alpha)$ for every $\alpha \in I$.

An element $w \in W$ is said to be a $\theta$-twisted involution if $\theta(w)=w^{-1}$.

Corollary 3.3 Every $m \in \mathcal{M}_{\theta}$ is both an involution and a $\theta$-twisted involution.

Proof Let $m \in \mathcal{M}_{\theta}$ and let the notation be as in Lemma 3.2. Then $m^{2}=w_{0} w_{0, I} w_{0, I} w_{0}=1$. Since $\theta(m)=m$, one also has $\theta(m)=m^{-1}$.

Definition 3.4 A subset $I$ of $\Gamma$ is said to have Property (1) if $I$ is both $\delta_{0}$ and $\theta$ invariant and if $\delta_{0} \theta(\alpha)=-w_{0, I}(\alpha)$ for all $\alpha \in I$.

By Lemma 3.2, every $m \in \mathcal{M}_{\theta}$ is of the form $m=w_{0} w_{0, I}$ for some $I \subset \Gamma$ with Property (1). Let $\langle$,$\rangle be the pairing on \Gamma$ induced from the Killing form of $\mathfrak{g}$. The following Definition 3.5 is inspired by [2, Lemma 4.1].

Definition 3.5 For a subset $I$ of $\Gamma$, an $\alpha \in I$ is said to be isolated if $\left\langle\alpha, \alpha^{\prime}\right\rangle=0$ for every $\alpha^{\prime} \in I \backslash\{\alpha\}$. A subset $I$ of $\Gamma$ is said to have Property (2) if for every isolated $\alpha \in I$, there is no $\beta \in \Gamma \backslash\{\alpha\}$ with the following properties

(a) $\langle\alpha, \alpha\rangle=\langle\beta, \beta\rangle$ and $\langle\beta, \alpha\rangle \neq 0$

(b) $\left\langle\beta, \alpha^{\prime}\right\rangle=0$ for all $\alpha^{\prime} \in I \backslash\{\alpha\}$;

(c) $\delta_{0} \theta(\beta)=\beta$.

Lemma 3.6 For every $m \in \mathcal{M}_{\theta}, I_{m}=\{\alpha \in \Gamma: m \theta(\alpha)=\alpha\} \subset \Gamma$ has Property (2). 
Proof Let $m \in \mathcal{M}_{\theta}$. Suppose that $\alpha \in I_{m}$ is isolated and that there exists $\beta \in \Gamma \backslash\{\alpha\}$ with properties (a), (b), and (c) in Definition 3.5. Let $I_{m}^{\prime}=I_{m} \backslash\{\alpha\}$. Since $\alpha \in I_{m}$ is isolated, $w_{0, I_{m}}=s_{\alpha} w_{0, I_{m}^{\prime}}$, so by (b) and (c), $m \theta(\beta)=w_{0, I_{m}} w_{0} \theta(\beta)=-s_{\alpha} w_{0, I_{m}^{\prime}}(\beta)=-s_{\alpha}(\beta)$, and

$$
s_{\alpha} s_{\beta} m s_{\theta(\beta)} s_{\theta(\alpha)}=s_{\alpha} s_{\beta} s_{m \theta(\beta)} m s_{\theta(\alpha)}=s_{\alpha} s_{\beta} s_{\alpha} s_{\beta} s_{\alpha} m s_{\theta(\alpha)} .
$$

By (a), $s_{\alpha} s_{\beta} s_{\alpha} s_{\beta} s_{\alpha}=s_{\beta}$, so $s_{\alpha} s_{\beta} m s_{\theta(\beta)} s_{\theta(\alpha)}=s_{\beta} m s_{\theta(\alpha)}=s_{\beta} s_{\alpha} m$. Since $m^{-1}(\alpha)=$ $\theta(\alpha)>0, l\left(s_{\beta} s_{\alpha} m\right) \geq l(m)$. Since $s_{\beta} s_{\alpha} m$ is in the same $\theta$-twisted conjugacy class as $m$, we have $s_{\beta} s_{\alpha} m=m$, or $s_{\alpha} s_{\beta}=1$, which is a contradiction.

\subsection{The classification of $m \in \mathcal{M}_{\theta}$}

For $\theta \in \operatorname{Aut}(\Gamma)$, let $\mathcal{I}_{\theta}$ be the collection of all subsets $I$ of $\Gamma$ that have Properties (1) and (2). Note that the empty set $\varnothing$ is always in $\mathcal{I}_{\theta}$. Also note that if $\theta \in \operatorname{Aut}(\Gamma)$ is not the identity automorphism, then $\Gamma$ does not have Property (1), so $\Gamma \notin \mathcal{I}_{\theta}$.

Proposition 3.7 (1) $\quad$ For $G=D_{4}$ and $\theta \in \operatorname{Aut}(\Gamma)$ of order $3, I \in \mathcal{I}_{\theta}$ if and only if $I=\emptyset$ or $I=\left\{\alpha_{2}\right\}$, where $\alpha_{2}$ is the simple root that is not orthogonal to any of the other three.

(2) For $G$ simple and $\theta \in \operatorname{Aut}(\Gamma)$ of order 2 , the list for $I \in \mathcal{I}_{\theta}$ is the same as that given in [10, Table 2], namely, either $I=\emptyset$ or I is one the following:

$A_{2 n}, n \geq 1, \theta=\delta_{0}$ : no non-empty $I$ in $\mathcal{I}_{\theta}$.

$A_{2 n+1}, n \geq 1, \theta=\delta_{0}: I=\left\{\alpha_{2 l+1}: 0 \leq l \leq n\right\}$.

$D_{4}: I=\left\{\alpha_{2}\right\} \cup \Gamma(2, \theta)$, where $\Gamma(2, \theta)$ is the $\theta$-orbit in $\Gamma$ with 2 elements.

$D_{2 n}, n>2, \theta\left(\alpha_{2 n-1}\right)=\alpha_{2 n}: I_{l}=\Gamma \backslash\left\{\alpha_{1}, \alpha_{2}, \ldots, \alpha_{2 l-1}\right\}$ for $1 \leq l \leq n-1$.

$D_{2 n+1}, n \geq 2, \theta=\delta_{0}: I_{l}=\Gamma \backslash\left\{\alpha_{1}, \alpha_{2}, \ldots, \alpha_{2 l-1}\right\}$ for $1 \leq l \leq n$.

$E_{6}, \theta=\delta_{0}: I=\left\{\alpha_{2}, \alpha_{3}, \alpha_{4}, \alpha_{5}\right\}$ with the simple roots labeled as

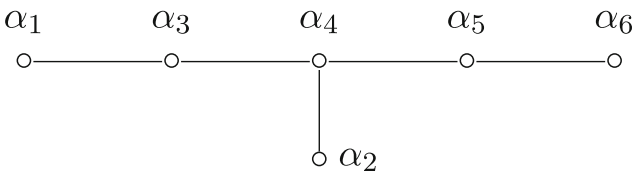

Proof (1) is easy to deduce and (2) is proved case-by-case. We omit the details.

By Lemma 3.2 and Lemma 3.6, we have, for $\theta \in \operatorname{Aut}(\Gamma)$, the well-defined map

$$
\psi: \mathcal{M}_{\theta} \longrightarrow \mathcal{I}_{\theta}: \quad m \longmapsto I_{m}=\{\alpha \in \Gamma: m \theta(\alpha)=\alpha\} .
$$

Since $m=w_{0} w_{0, I_{m}}$ for every $m \in \mathcal{M}_{\theta}$, the map $\psi$ is injective.

Assume now $\theta \in \operatorname{Aut}^{\prime}(G)$, and let $\widetilde{\mathcal{M}}_{\theta} \subset W$ be defined as in (1.2). By Remark 1.2, $\widetilde{\mathcal{M}}_{\theta}$ depends only on the corresponding $\theta \in \operatorname{Aut}(\Gamma)$. Let $\widetilde{\psi}: \widetilde{\mathcal{M}}_{\theta} \rightarrow \mathcal{I}_{\theta}$ be the restriction of $\psi$ to $\widetilde{\mathcal{M}}_{\theta} \subset \mathcal{M}_{\theta}$.

Theorem 3.8 For $G$ simple and $\theta \in \operatorname{Aut}^{\prime}(G)$ an outer automorphism of $G$, the map $\widetilde{\psi}$ : $\widetilde{\mathcal{M}}_{\theta} \rightarrow \mathcal{I}_{\theta}$ is bijective. Consequently,

$$
\widetilde{\mathcal{M}}_{\theta}=\mathcal{M}_{\theta}=\left\{w_{0} w_{0, I}: I \in \mathcal{I}_{\theta}\right\} .
$$

Proof It is enough to prove that $\tilde{\psi}$ is surjective, and we may assume that $G$ is adjoint.

First assume that $\theta \in \operatorname{Aut}(\Gamma)$ has order 2, and let $I \in \mathcal{I}_{\theta}$. By Proposition 3.7, $I$ is in [10, Table 2], so $\left(I, \delta_{0} \theta\right)$ is admissible in the sense of [10, No. 2.2]. By [10, No. 4 and No. 5], there exists $h \in H$ such that $\operatorname{Ad}_{h} \theta \in \operatorname{Aut}(G)$ is an involution and that $w_{0} w_{0, I}=m_{C}$, where $C$ is the $\theta$-twisted conjugacy class through $h$. In particular, $w_{0} w_{0, I} \in \widetilde{\mathcal{M}}_{\theta}$. 
It remains to consider the case of $G=D_{4}$ with $\theta \in \operatorname{Aut}(\Gamma)$ having order 3 . It is clear that $w_{0}=m_{C}$ if $C$ is the $\theta$-twisted conjugacy class of $\dot{w}_{0}$, so $w_{0} \in \overline{\mathcal{M}}_{\theta}$. We only need to show that $w_{0} s_{2} \in \widetilde{\mathcal{M}}_{\theta}$. To this end, we may, by Remark 1.2, assume that $\theta \in \operatorname{Aut}^{\prime}(G)$ is a diagram automorphism of $G$ in the sense that $\theta \circ x_{\alpha}=x_{\theta \alpha}$ for $\alpha \in \Gamma$, where for each $\alpha \in \Gamma, x_{\alpha}: \mathbf{k}_{a} \rightarrow G$ is a fixed choice of one-parameter root subgroup corresponding to $\alpha$. In particular, $\theta^{3}=\operatorname{Id}_{G}$. Let $C_{e}$ be the $\theta$-twisted conjugacy class through the identity element $e$ of $G$. It is well-known that $\mathfrak{g}^{\theta}$ is of type $G_{2}$ [8, Chapter 24] so it is 14-dimensional. Thus

$$
\operatorname{dim} C_{e}=\operatorname{dim} G-14=14=l\left(w_{0} s_{2}\right)+\operatorname{rk}\left(1-w_{0} s_{2} \theta\right) .
$$

Since $l\left(w_{0}\right)+\operatorname{rk}\left(1-w_{0} \theta\right)=16$, we know by Lemma 2.1 that $m_{C_{e}} \neq w_{0}$ so $m_{C_{e}}=w_{0} s_{2}$. In particular, $w_{0} s_{2} \in \widetilde{\mathcal{M}}_{\theta}$ and $C_{e}$ is spherical. See $[6, \S 4.5]$ for another proof of the fact that $w_{0} s_{2} \in \widetilde{\mathcal{M}}_{\theta}$ and that $C_{e}$ is spherical.

Example 3.9 Let $G=D_{4}$ be of adjoint type, and let $\theta \in \operatorname{Aut}^{\prime}(G)$ be a triality automorphism of $G$ as in the proof of Theorem 3.8. Since $l\left(w_{0} s_{2}\right)+\operatorname{rk}\left(1-w_{0} s_{2} \theta\right)=14$ and $l\left(w_{0}\right)+\operatorname{rk}\left(1-w_{0} \theta\right)=16, \operatorname{dim} C \geq 14$ for every $\theta$-twisted conjugacy class $C$ in $G$, and, by Theorem 1.1, $\operatorname{dim} C=14$ or 16 if $C$ is spherical.

Recall from [11] that a $\theta$-twisted conjugacy class is semisimple if it contains an element in $H$. For $h \in H$, let $C_{h} \subset G$ be the $\theta$-twisted conjugacy class of $h$. Label the simple roots as $\Gamma=\left\{\alpha_{j}: 1 \leq j \leq 4\right\}$ such that $\theta\left(\alpha_{2}\right)=\alpha_{2}, \theta\left(\alpha_{1}\right)=\alpha_{3}, \theta\left(\alpha_{3}\right)=\alpha_{4}$, and $\theta\left(\alpha_{4}\right)=\alpha_{1}$. We now show that if $h^{\alpha_{2}}=h^{\alpha_{1}} h^{\alpha_{3}} h^{\alpha_{4}}=1$, then $m_{C_{h}}=w_{0} s_{2}$ and $C_{h}$ is spherical, and otherwise, $m_{C_{h}}=w_{0}$ and $\operatorname{dim} C_{h} \geq 20$, so $C_{h}$ is not spherical. Here, for a character $\mu$ on $H$, $h^{\mu}$ denotes the value of $\mu$ on $h$.

Label the positive roots in $\Delta_{+} \backslash \Gamma$ as

$$
\begin{aligned}
\alpha_{5} & =\alpha_{1}+\alpha_{2}, \quad \alpha_{6}=\alpha_{2}+\alpha_{3}, \quad \alpha_{7}=\alpha_{2}+\alpha_{4}, \\
\alpha_{8} & =\alpha_{1}+\alpha_{2}+\alpha_{3}, \quad \alpha_{9}=\alpha_{2}+\alpha_{3}+\alpha_{4}, \quad \alpha_{10}=\alpha_{1}+\alpha_{2}+\alpha_{4}, \\
\alpha_{11} & =\alpha_{1}+\alpha_{2}+\alpha_{3}+\alpha_{4}, \quad \alpha_{12}=\alpha_{1}+2 \alpha_{2}+\alpha_{3}+\alpha_{4} .
\end{aligned}
$$

Then $\left\{\alpha_{1}, \alpha_{3}, \alpha_{4}\right\},\left\{\alpha_{5}, \alpha_{6}, \alpha_{7}\right\}$ and $\left\{\alpha_{8}, \alpha_{9}, \alpha_{10}\right\}$ are the three $\theta$-orbits in $\Delta_{+}$of size 3 and $\theta\left(\alpha_{11}\right)=\alpha_{11}$ and $\theta\left(\alpha_{12}\right)=\alpha_{12}$. Note that the sets $\left\{\alpha_{1}, \alpha_{3}, \alpha_{4}, \alpha_{12}\right\},\left\{\alpha_{5}, \alpha_{6}, \alpha_{7}, \alpha_{11}\right\}$, and $\left\{\alpha_{8}, \alpha_{9}, \alpha_{10}, \alpha_{2}\right\}$ consist of orthogonal roots, and, with $s_{j}$ denoting the reflection in $W$ defined by $\alpha_{j}$ for $1 \leq j \leq 12, w_{0}=s_{1} s_{3} s_{4} s_{12}=s_{5} s_{6} s_{7} s_{11}=s_{8} s_{9} s_{10} s_{2}$.

Recall that the stabilizer subalgebra of $\mathfrak{g}$ at $h$ is $\mathfrak{g}_{h}=\mathfrak{g}^{\operatorname{Ad}_{h} \theta}$. Since $\operatorname{dim} \mathfrak{h}^{\operatorname{Ad}_{h} \theta}=\operatorname{dim}$ $\mathfrak{h}^{\theta}=2$, one has $\operatorname{dim} \mathfrak{g}_{h}=2+2 n$, where $n=\#\left\{i \in\{1,2,5,8,11,12\}: \lambda_{i}(h)=1\right\}$, with $\lambda_{i}(h)=h^{\alpha_{i}+\theta\left(\alpha_{i}\right)+\theta^{2}\left(\alpha_{i}\right)}$ for $i \in\{1,5,8\}$ and $\lambda_{i}(h)=h^{\alpha_{i}}$ for $i \in\{2,11,12\}$. Let $\Lambda(h)=\left\{\lambda_{i}(h): i \in\{1,2,5,8,11,12\}\right\}$. Then $\lambda_{1}(h)=h^{\alpha_{1}} h^{\alpha_{3}} h^{\alpha_{4}}, \lambda_{2}(h)=h^{\alpha_{2}}$, and

$$
\Lambda(h)=\left\{\lambda_{1}(h), \lambda_{2}(h), \lambda_{1}(h)\left(\lambda_{2}(h)\right)^{3},\left(\lambda_{1}(h)\right)^{2}\left(\lambda_{2}(h)\right)^{3}, \lambda_{1}(h) \lambda_{2}(h), \lambda_{1}(h)\left(\lambda_{2}(h)\right)^{2}\right\} .
$$

Case $1 h^{\alpha_{2}}=h^{\alpha_{1}} h^{\alpha_{3}} h^{\alpha_{4}}=1$. In this case, $n=6$, $\operatorname{dim} \mathfrak{g}_{h}=14$, and $\operatorname{dim} C_{h}=28-14=$ 14. It follows from Lemma 2.1 that $C_{h}$ is spherical and $m_{C_{h}}=w_{0} s_{2}$. Note that in this case, $\left(\operatorname{Ad}_{h} \theta\right)^{3}=\operatorname{Id}_{G}$, so $\mathfrak{g}_{h}$ is again of type $G_{2}$.

Case $2 h^{\alpha_{2}} \neq 1$ or $h^{\alpha_{1}} h^{\alpha_{3}} h^{\alpha_{4}} \neq 1$. In this case, $n \leq 5$. In fact, it is easy to see that one can not have $n=5$ nor $n=4$, so $n \leq 3$, and $\operatorname{dim} C_{h}=28-\operatorname{dim} \mathfrak{g}_{h} \geq 20$. Thus $C_{h}$ is not spherical. We use the approach in $[6, \S 4.5]$ to prove that $m_{C_{h}}=w_{0}$. First assume that $h^{\alpha_{2}} \neq 1$. Fix a one-parameter root subgroup $x_{\alpha}: \mathbf{k}_{a} \rightarrow G$ for $\alpha \in-\left\{\alpha_{8}, \alpha_{9}, \alpha_{10}\right\}$ such that $\theta \circ x_{\alpha}=x_{\theta(\alpha)}$ for every $\alpha \in-\left\{\alpha_{8}, \alpha_{9}, \alpha_{10}\right\}$ (recall that $\theta^{3}=\operatorname{Id}_{G}$ ). For $a, b, c, d \in \mathbf{k} \backslash\{0\}$, let $g=x_{-\alpha_{2}}(a) x_{-\alpha_{8}}(b) x_{-\alpha_{9}}(c) x_{-\alpha_{10}}(d) \in G$. Then

$$
g h \theta(g)^{-1}=x_{-\alpha_{2}}\left(a-h^{-\alpha_{2}} a\right) x_{-\alpha_{8}}\left(b-h^{-\alpha_{8}} d\right) x_{-\alpha_{9}}\left(c-h^{-\alpha_{9}} b\right) x_{-\alpha_{10}}\left(d-h^{-\alpha_{10}} c\right) h .
$$


Choosing $a, b, c, d$ such that $a \neq 0, b-h^{-\alpha_{8}} d \neq 0, c-h^{-\alpha_{9}} b \neq 0$ and $d-h^{-\alpha_{10}} c \neq 0$, one has $g h \theta(g)^{-1} \in C_{h} \cap\left(B w_{0} B\right) \cap B_{-}$, so $m_{C_{h}}=w_{0}$. If $h^{\alpha_{2}}=1$, then $h^{\alpha_{1}} h^{\alpha_{3}} h^{\alpha_{4}} \neq 1$. In this case, $h^{\alpha_{11}}=h^{\alpha_{12}} \neq 1$. Using the fact $w_{0}=s_{5} s_{6} s_{7} s_{11}$ or the fact $w_{0}=s_{1} s_{3} s_{4} s_{12}$ and arguments similar to the above, one sees that $m_{C_{h}}=w_{0}$.

Remark 3.10 Our interest in the dimension formula (1.1) comes from Poisson geometry: by a general construction in [7], an automorphism $\theta$ of $G$ naturally induces a Poisson structure $\pi_{\theta}$ on $G$ such that each $\theta$-twisted conjugacy class is a Poisson submanifold with respect to $\pi_{\theta}$. For a $\theta$-twisted conjugacy class $C$ in $G$, the element $m_{C}$ is closely related to the smallest dimension of the symplectic leaves of $\pi_{\theta}$ in $C$, and formula (1.1) is closely related to the vanishing of $\pi_{\theta}$ in $C$. These results and a detailed study of the Poisson structure $\pi_{\theta}$ will appear elsewhere.

Acknowledgments The author would like to thank G. Carnovale, K. Y. Chan and G. Lusztig for discussions. The author is especially grateful to G. Carnovale and M. Costantini for pointing out some of the results in [6]. She would also like to thank the referee for very helpful comments. Research in this paper was partially supported by HKRGC grants 703405 and 703707 and by the Seed Fund for Basic Research from the HKU (Project code 10400942).

Open Access This article is distributed under the terms of the Creative Commons Attribution Noncommercial License which permits any noncommercial use, distribution, and reproduction in any medium, provided the original author(s) and source are credited.

\section{References}

1. Cantarini, N., Carnovale, G., Costantini, M.: Spherical orbits and representations of $\mathcal{U}_{\epsilon}(\mathfrak{g})$. Trans. Groups 10(1), 29-62 (2005)

2. Carnovale, G.: Spherical conjugacy classes and involutions in the Weyl group. Math. Z. 260(1), 1-23 (2008)

3. Chan, K.Y., Lu, J-H., To, S.: On intersections of conjugacy classes and Bruhat cells. Trans. Groups 15(2), 243-260 (2010)

4. Chan, K.Y.: Weyl group elements associated to conjugacy classes. MPhil thesis in Mathematics, The University of Hong Kong (2010)

5. Costantini, M.: On the coordinate ring of spherical conjugacy classes. Math. Z. 264, 327-359 (2010)

6. Costantini, M.: A classification of unipotent conjugacy classes in bad characteristics. arXiv:0906.5063

7. Lu, J.-H., Yakimov, M.: Group orbits and regular partitions of Poisson manifolds. Comm. Math. Phys 283(3), 729-748 (2008)

8. Porteous, I.: Clifford Algebras and the Classical Groups. Cambridge University Press, Cambridge (1995)

9. Springer, T.A.: Some results on algebraic groups with involutions, algebraic groups and related topics. Adv. Stud. Pure Math. 6, 525-543 (1985)

10. Springer, T.A.: The classification of involutions of simple algebraic groups. J. Fac. Sci. Univ. Tokyo Sect. IA Math. 34, 655-670 (1987)

11. Springer, T.A.: Twisted conjugacy in simply connected groups. Trans. Groups 11(3), 539-545 (2006) 